\title{
Analysis of the Early Clinical Effect of Simultaneous Ipsilateral Total Hip and Knee Arthroplasty in the Treatment of End-stage Hemophilic Arthritis
}

\section{Xu Peng}

Second Affiliated Hospital of Chongqing Medical University

Shirong Chen ( $\nabla$ cqjoint@hotmail.com )

Second Affiliated Hospital of Chongqing Medical University

\section{Xiangjun Cheng}

Second Affiliated Hospital of Chongqing Medical University

Xiaowei Xie

Second Affiliated Hospital of Chongqing Medical University

Mao Nie

Second Affiliated Hospital of Chongqing Medical University

\section{Research Article}

Keywords: hemophilic arthritis, total hip arthroplasty, total knee arthroplasty, coagulation factor

Posted Date: December 10th, 2021

DOI: https://doi.org/10.21203/rs.3.rs-1143714/v1

License: (9) This work is licensed under a Creative Commons Attribution 4.0 International License. Read Full License 


\section{Abstract}

Objective

To evaluate the clinical efficacy of simultaneous ipsilateral total hip arthroplasty (THA) and total knee arthroplasty (TKA) in the treatment of advanced hemophilic arthritis.

Method

the clinical data of 8 patients with advanced hemophilic arthritis, with ages of $31.6 \pm 6.2$ years (ranging from 25-38 years, who underwent ipsilateral THA and TKA at the ** Hospital from January 2018 to May 2021 were retrospectively analyzed. There were 6 cases of hemophilia A and 2 cases of hemophilia B. The preoperative level of coagulation factor VIII was $1.8( \pm 1.3) \%$ activated partial prothrombin time (aPTT) was $131.2( \pm 35.3) \mathrm{s}$. A comparison of the Harris score before and 0.3-1 year after operation was performed to evaluate hip function, American hospital for special surgery (HSS) knee score and American Knee society score (KSS) score.

Result

During the follow-up period of $1.4( \pm 1.1)$ years, no intra-articular bleeding and skin dehiscence were found in the early postoperative period, and no joint infection, bleeding, prosthesis loosening and sinking were found in the last follow-up visit. The Harris score increased from $16.8( \pm 4.4)$ preoperatively to 77.6 $( \pm 7.1)$ postoperatively, HSS knee score increased from $41.8( \pm 4.2)$ preoperatively to $76.0( \pm 5.8)$ postoperatively, the clinical KSS increased from $35.6( \pm 10.8)$ preoperatively to $79.2( \pm 6.9)$ postoperatively. The KSS increased from $22.8( \pm 8.4)$ preoperatively to $72.0( \pm 5.9)$ postoperatively at the last follow-up visit. The differences were statistically significant $(P<0.05)$.

\section{Conclusions}

Ipsilateral THA and TKA in the treatment of advanced hemophilic arthritis can effectively relieve pain, and improve hip and knee joint function, as well as the quality of life, and can thus be recommended as a safe and effective measure for the treatment of advanced hemophilic arthritis.

\section{Background}

Hemophilia is a hereditary hemorrhagic disease caused by deficiency of coagulation factors. It can be divided into hemophilia A (deficiency of coagulation factor VIII) and hemophilia B (deficiency of coagulation factor IX), which are mostly transmitted from female carriers to male offspring[1]. Hemophilia patients are prone to bleeding in joints, muscles and deep tissues from a young age, especially in large joints that are easy to be injured or overloaded[2]. Hemophilic arthritis (HA) is one of the major complications of hemophilia, which can cause joint contracture, deformity and pain, which ultimately lead to obvious limitation or impairment of joint function, which seriously affects the quality of life of patients. The incidence rate of HA in descending rank order is knee, elbow and hip joints[3]. 
Although prophylactic coagulation factor arthroplasty therapy can significantly reduce the bleeding frequency, it cannot completely prevent the progression of joint disease in hemophilia patients[4]. As an important method of HA treatment, surgical intervention can greatly improve the quality of life of patients, which is particularly of great importance. Due to the individuality of HA patients, age structure, severe preoperative joint deformity, intraoperative and postoperative bleeding and hemostasis and other problems, the surgical treatment of HA is much more difficult than that of general patients. With the use of coagulation factor concentrate, total joint arthroplasty has become the main method for the treatment of advanced type A HA, which can significantly improve joint function, correct joint deformation, reduce pain and improve the long-term quality of life of patients[5], However, since patients usually have multiple joint issues, this treatment methods can be expensive due to the high cost of coagulation factors. Total hip arthroplasty (THA) and total knee arthroplasty (TKA) can significantly relieve pain, improve joint function, lower the disability rate and reduce treatment cost. However, the benefits and risks of simultaneous ipsilateral THA and TKA in patients with advanced hemophilic knee arthritis are still controversial, and there are few relevant reports in the literature[6] \. From January 2018 to May 2021, we treated 8 patients with advanced HA with simultaneous ipsilateral total hip and total knee arthroplasty, and achieved good clinical outcomes.

\section{Clinical Data}

\subsection{General information}

The 8 patients included in this study were male, 25-38 years of age (mean 31.6 years). There were 6 cases of hemophilia A and 2 cases of hemophilia B. They all had a history of hematology diagnosis and treatment and coagulation factor infusion. All patients were routinely tested for 4 coagulation factors, factor VIII and IX inhibitors and 10 immunizations (hepatitis, syphilis and HIV antibody tests were negative). The average activity of factor VIII in the 6 patients with type A hemophilia was $2.1( \pm 2.3) \%$, and that of factor IX in the 2 patients with type B hemophilia was $1.5( \pm 1.4) \%$. Preoperative factor VIII inhibitor test of all patients with type A hemophilia, and factor IX inhibitor test of all patients with type B hemophilia were zero. All patients had different degrees of ipsilateral knee and hip pain and decreased range of motion, as well as different degrees of joint deformity, which seriously affected their quality of life. Conservative treatment was ineffective. Six patients (6 knees) had genu varus deformity (10-25 degrees), and two patients ( 2 knees) had genu valgus deformity (12-20 degrees).

Inclusion criteria: all patients had hemophilic ipsilateral hip and knee arthritis. Patients with hemophilia whose hip joint function was clearly impaired and whose knee joint was classified as stage IV or V according to Arnold Hilgartner imaging classification and had no factor VIII or factor IX inhibitor before operation. All patients received informed consent to about the treatment plan and their participation was approved by the hospital ethics committee.

Exclusion criteria: joint infection; hip and knee arthroplasty was performed on the other side or at the same time; osteoarthritis, rheumatoid arthritis, etc.; the detection of factor VIII inhibitor in type A 
hemophilia was positive, and the detection of factor IX inhibitor in type B hemophilia was positive.

\subsection{Perioperative coagulation factor arthroplasty therapy}

In the perioperative period of THA and TKA, the monitoring of coagulation factor arthroplasty therapy is mainly performed by measuring activated partial thromboplastin time (aPTT), the activity and concentration of coagulation factors, and the presence of coagulation factor inhibitors [7]. After admission, patient is asked to consult with hematology department and schedule tests. For patients with type A hemophilia: the keyuqi recombinant factor VIII preparation (Bayer AG, Leverkusen, Germany) was used at the supplementary dose of $(U)=$ body weight $(\mathrm{kg}) \times($ required factor : C level\% - actual factor : C level\%) / 2. The half-life of factor VIII is 8-12 h, so half of the first dose should be

supplemented every 8-12 $\mathrm{h}$ to maintain the level of factor VIII. Blood coagulation factor was infused at a dose of $25 \mathrm{IU} / \mathrm{kg}$ every 8-12 h 1-3 days after operation to keep its activity at 60-80\%. Factor was infused every 8-12 h at a dose of $20 \mathrm{IU} / \mathrm{kg} \mathrm{4-7} \mathrm{days} \mathrm{after} \mathrm{operation,} \mathrm{and} \mathrm{its} \mathrm{activity} \mathrm{was} \mathrm{maintained} \mathrm{at} 50$ - $60 \%$. At 1-2 weeks after operation, factor was infused intravenously at the dose of $20 \mathrm{lU} / \mathrm{kg}$ to maintain the plasma factor : C level of $30-50 \%$. Subsequently, according to the bleeding situation and factor : C level, the amount of coagulation factor supplement was reduced until discharge.

For patients with type B hemophilia B: The Pfizer's bFGF IX preparation (Pfizer Inc., New York, NY, USA) was used at the supplementary dose $(U)=$ body weight $(\mathrm{kg}) \times($ required factor IX: C level\% - actual factor IX: C level\%). The half-life of factor IX is 12-24 h, so it is given 1-2 times a day. Blood coagulation factor IX was infused at a dose of $25 \mathrm{IU} / \mathrm{kg}$ every $12-24 \mathrm{~h} \mathrm{1-3}$ days after operation to keep its activity at $60-80 \%$. Factor IX was infused every 12-24 h at a dose of $20 \mathrm{IU} / \mathrm{kg}$ 4-7 days after operation, and its activity was maintained at $50-60 \%$. At 1-2 weeks after operation, factor IX was infused intravenously at the dose of $20 \mathrm{IU} / \mathrm{kg}$ to maintain the plasma factor IX: C level of $30-50 \%$. Subsequently, according to the bleeding situation and factor IX: C level, the amount of coagulation factor supplement was reduced until discharge.

\subsection{Operation method}

In this study, after general anesthesia, all patients were first operated on the hip and then the knee.

For THA: the patient was positioned in the healthy lateral position, and the surgical field was routinely disinfected and covered with towel. The posterolateral incision of the affected hip, about 10-12 cm long, was made to cut the skin and subcutaneous adipose tissue, and the fascia lata surface was properly stripped to form a "moving window" (also conducive to close the incision tightly). The fascia lata (gluteal fascia) was cut along the posterior margin of the trochanter with an electric knife, and then the gluteus maximus fiber bundle was separated using the electric knife to expose the posterior outer fat layer of the joint capsule. After confirming the posterior edge of the gluteus medius and gluteus minimus, the affected limb was slightly rotated, the insertion point of the piriformis was peeled off along the piriformis fossa at the posterior edge of the trochanter, a "U" shape incision was made in the joint capsule to reach the lateral apex of the acetabulum, and expose the femoral neck and head. The affected limb was 
straightened in the neutral position, and marked with an electric knife at the midpoint of the greater trochanter. A single suture was placed and fixed on the skin of the iliac crest along the long axis of the limb. The operator pulled the suture to the marked position with appropriate tension, cut and retained it, so as to evaluate whether the limb length was balanced after the prosthesis implantation. The hip dislocation was caused by extreme internal rotation and adduction of the femur. The femoral neck osteotomy was protected by an acetabular retractor with $90^{\circ}$ internal rotation of the affected limb, $45^{\circ}$ hip flexion and $90^{\circ}$ knee flexion. According to the preoperative $X$-ray measurement results, the femoral neck osteotomy was performed with a femoral distance of 1-1.5 cm reserved, and the femoral head was removed with a head extractor. The acetabulum was exposed step by step and the labrum was cleaned by using the acetabular retractor of minimally invasive surgery (MIS). The thickened joint capsule, osteophyte and round ligament residue were removed until the bone acetabulum edge was completely exposed. First, the acetabulum file with the size of the femoral head was selected to grind the acetabulum to the bottom of the horseshoe fossa of the acetabulum, and then the grinding file was in turn increased to completely remove the cartilage surface around the acetabulum, resulting in punctate bleeding, especially after the weight-bearing area at the top of the acetabulum. Then, the non-cemented acetabulum was placed under the protection of packaging film (to prevent soft tissue embedding). After confirming the proper position of the prosthesis, the cup was hammered and pressed. The hook needle was used to determine whether the interface between the cup and the bone was tightly pressed through the screw hole, and whether there was soft tissue embedded in the edge of the cup. After the press fit was satisfactory, titanium screws were selected according to the patient's age and bone condition. Some patients with excellent bone and ideal press fit were fixed without screws. For the patients with obvious limitation of hip joint movement before operation, it was necessary to use a MIS hook for a reasonable exposure, making full use of the "mobile window" to gradually release the peri hip, especially the anterior and medial adhesion scar tissue and joint capsule, and gradually increase the range of motion. If there was bone defect, autogenous femoral head or allogeneic cancellous bone graft was used, and acetabular prosthesis was implanted after the preparation of the bone acetabulum was satisfactory. Then, the ceramic liner was implanted along the direction of the prosthesis to avoid soft tissue embedding. The knee was bent and the femur was rotated $90^{\circ}$ so that the leg was perpendicular to the operating table. The proximal femur was lifted with a "Jaw" retractor, and the acetabular retractor was placed in the greater and lesser trochanter of the femur. After determining the anteversion angle of $15-20^{\circ}$, the box osteotome slotter was used to slot and ream the pulp manually along the direction of the femoral anterior arch (, and the proximal femur was ground with a cone-shaped grinding drill. After reaming to the appropriate model in turn, the femoral stem test mold and test mold head were placed and the hip joint was reset. The range of motion and stability of the hip joint were tested (hip flexion $>100^{\circ}$, hip extension $>10^{\circ}$ and internal rotation $>60^{\circ}$ ), and the prosthesis was placed after the length of both lower limbs was balanced (the distance between the end of the skin suture and the greater trochanter mark was increased, compared with the preoperative X-ray template measurement value and the comprehensive evaluation of solid measurement). A large amount of normal saline was used to thoroughly wash and stop bleeding. After checking the instruments and gauze, a normal pressure drainage tube (open after clamping for $6 \mathrm{~h}$ ) was placed and sutured layer by layer and bandaged. 
Then, after the patient was placed in the supine position, the tourniquet was tied at the root of the affected thigh, and the affected limb was routinely disinfected and covered. The anterior patellar incision made in the affected knee joint was about $12 \mathrm{~cm}$ in length. The skin, subcutaneous tissue and fascia were cut in turn, and electrocoagulation was used to stop bleeding. The tibial plateau and femoral condyle of the knee joint were exposed, the patella was turned over to the outside, and the fibrous tissue of the patella was removed with an electric knife. Due to the severe deformity of the femoral condyle and tibial plateau, complete loss of joint space, severe congestion and edema of the synovium, the force line of the lower limb was preliminarily determined using a vertical line rod. The tibial plateau is surrounded by a large number of proliferative synovial tissue and osteophytes. Therefore, the medial and lateral sides of the tibial plateau were separately released. The osteophytes on the femoral condyle and tibial plateau were removed with bone biting forceps to fully expose the tibial plateau. After checking that the knee joint flexion was 90 degrees, a hole in front of the attachment point of the posterior cruciate ligament of the distal femoral intercondylar fossa was drilled, the pulp content was drained, and the anteroposterior femoral dimension measurement guide into the hole.

Methods: the adhesive tissue around the medial and lateral sides of the femoral condyle is released, the hyperplastic osteophyte is removed, proximal tibial osteotomy is performed, the medial and lateral center of the tibia are located, the alignment system of the extramedullary osteotomy guide is installed and safely fixed, the osteotomy plate and extramedullary locator are placed on the tibia, the rod is paralleled with the medial tibial muscle, and the angle of the guide is adjusted, so that the body of the guide is parallel to the anterior tibial shaft. The position of the cutting plate was measured with a tibial osteotomy thickness gauge. The osteotomy plate was nailed to the tibia, and the tibial plateau was cut along the osteotomy device with a pendulum saw. The osteotomy was performed at the back of the femur, the bone defect of the lateral tibia was filled with the osteotomy bone graft, and the suitable tibial pad was placed. The knee joint was straightened to be able to see good flexion and extension. Analgesic drugs (morphine hydrochloride injection $8 \mathrm{mg}$, morphine hydrochloride injection $8 \mathrm{mg}$, morphine hydrochloride injection 4 mg, morphine hydrochloride injection $8 \mathrm{mg}$ ) were injected into the posterior capsule, quadriceps femoris tendon and around the incision, respectively. Ropivacaine $40 \mathrm{mg}$, Diprospan $1 \mathrm{mg}$, mixed with normal saline $(60 \mathrm{ml}$ in total) were also given. The femoral and tibial bone cement prostheses were correspondingly installed. A large amount of normal saline was used to thoroughly wash and stop bleeding. After checking the instruments and gauze, a normal pressure drainage tube (open after clamping for $6 \mathrm{~h}$ ) was placed and sutured layer by layer, and pressure bandaged.

All patients were given tranexamic acid before operation. Tranexamic acid is an antifibrinolytic drug that inhibits the activation of plasminogen. As an antifibrinolytic drug, tranexamic acid can reduce blood loss and transfusion demand in patients with hemophilia. It has good safety and effectiveness, and has no thromboembolic events or other adverse reactions. Cefazolin sodium powder $(1.0 \mathrm{~g})$ injection (Shandong Lukang Pharmaceutical Co., Ltd., Jining City, China) was given by intravenous drip $0.5 \mathrm{~h}$ before operation.

\subsection{Postoperative management}


After the operation, according to the supplementary dose requirements for calculating the coagulation factor activity, the recombinant human coagulation factor VIII was infused into hemophilia type A patients, and recombinant human coagulation factor IX powder injection was infused into hemophilia type B patients to maintain the activity of plasma factor VIII. The wound was constantly iced, and the postoperative analgesia pump was generally used for $48 \mathrm{~h}$. The drainage tube was removed after the drainage volume was less than $50 \mathrm{ml}$ the previous day. Foot pump exercise and pressure therapy were used to prevent postoperative deep vein thrombosis (DVT) without anticoagulation. The wound dressings were changed every day for the first 3 days after operation, and then every two days until the stitches were removed 2 weeks after operation. After the operation, the patient returned to the ward and placed in the supine position. The affected limb was abducted in the neutral position. A trapezoidal pillow was placed between the legs. The patient wore t-shoes in the affected foot to prevent external rotation. The affected limb was raised. A soft pillow was placed at the middle and lower 1 / 3 of the leg to make the knee joint fixed and straight. One or two days after the operation, we strengthened the isometric contraction of the quadriceps femoris, ankle flexion and extension, heel sliding, passive knee extension and elevation, and walking aids. Three or four days after the operation, knee extension and flexion exercises were performed along the edge of the bed. After discharge, the patients continued to perform isometric contraction of the quadriceps femoris and active knee flexion and extension exercises. The routine blood tests, including four coagulation items and the concentration of coagulation factors, were monitored, and the input of coagulation factors was timely adjusted. Tranexamic acid $(1 \mathrm{~g})$ twice a day was given within 1 week after operation. The patients were followed up at 6 weeks, 3 months, 6 months, 1 year and every year after the surgery. The clinical function and imaging were evaluated at each visit.

\subsection{Postoperative observation index}

The intraoperative blood loss, the decreasing trend of postoperative hemoglobin and the postoperative drainage of knee joint were examined. The incision was observed early after operation. The anteroposterior and lateral X-ray images of the knee joint at 1.5, 3, 6 and 12 months after operation were used to evaluate the loosening of the prosthesis. The Harris score, American hospital for special surgery (HSS) knee score and American knee society score (KSS) were recorded and compared before and 0.3-1 year after operation. During the follow-up, the X-ray images were examined to detect the signs of implant loosening. If the implant migration or dissolution $>2 \mathrm{~mm}$ was detected in the interface, the implant was designated as loosening.

\subsection{Data statistics}

The SPSS 25.0 software (IBM Corp., Armonk, NY, USA) was used to analyze the data. The measurement data were expressed as the mean \pm standard deviation( $\pm s$ ). A paired t-test was used for continuous variables and normal distribution data. A nonparametric test was used to compare continuous variables with non-normal distribution and the $\chi^{2}$ test was used to compare categorical variables. $P<0.05$ for the difference was considered statistically significant. 


\section{Results}

All 8 patients were followed up for longer than a year. The intraoperative blood loss was $(550 \pm 50) \mathrm{ml}$, the postoperative hemoglobin content was $(105.1 \pm 16.5) \mathrm{g} / \mathrm{L}$, and the postoperative drainage volume was $(525.5 \pm 75.5) \mathrm{ml}$. The level of factor : C increased from $1.5 \pm 1.3 \%$ preoperatively to $118.6 \pm 12.8 \%$ on the 1 st day postoperatively; the level of factor : C increased from $1.2 \pm 1.19 \%$ preoperatively to $106.1 \pm$ $12.8 \%$ on the 1 st day postoperatively $(P<0.05)$. Specifically, the 8 patients were followed up for $(1.4 \pm$ 1.1) years. There was no intra-articular bleeding, skin dehiscence, DVT, aseptic loosening and sinking of prosthesis at the last follow-up visit. Typical cases are shown in Figure 1.

Hip and knee function score: the Harris score increased from $16.8 \pm 4.4$ preoperatively to $77.6 \pm 7.1$ postoperatively, the HSS knee score increased from $41.8 \pm 4.2$ preoperatively to $76.0 \pm$ 5.8)postoperatively, the KSS clinical score increased from $35.6 \pm 10.8$ preoperatively to $79.2 \pm 6.9$ postoperatively, the KSS functional score increased from $22.8 \pm 8.4$ preoperatively to $72.0 \pm 5.9$ postoperatively at the last follow-up visit. The differences were statistically significant (all $P<0.05$; Table 1). There were no adverse reactions, such as infection, allergic reaction, immune reaction and rejection.

\section{Discussion}

To gain a better understanding of the pathogenesis of HA is critical for the diagnosis and treatment of this disease. Joint bleeding is the initiating factor in the pathogenesis of HA. After acute joint hemorrhage, it takes about one week for type A cells in the synovial intima, composed of macrophage like cells, to clear the blood from the joint cavity [7]. Since hemophilia patients are prone to recurrent intraarticular bleeding, the amount of intra-articular bleeding far exceeds the level of synovial tissue clearance, resulting in intra-articular hematocele and deposition of blood degradation metabolites in the joint[8]. Animal studies have shown that early intra-articular bleeding is positively correlated with HA disease progression[9]. In particular, the frequency of joint bleeding was positively correlated with the severity of coagulation factor deficiency in patients with hemophilia. The probability of joint bleeding in patients with hemophilia A was higher than that in patients with hemophilia B. Some studies have shown that $90 \%$ of hemophiliacs develop HA in the second and third decade of their lives $[4,10]$

Total joint arthroplasty is the gold standard for the treatment of advanced hemophilic hip and knee arthritis. Carulli et al. and Liddle et al.[6,11] reported 23 patients with hemophilic hip arthritis who were treated with THA, with an average follow-up of 8.4 years. When discharged, all patients were able to walk with full weight. The average preoperative hemophilia joint health score (HJHS) of the patients was 12.5 (range: 10-22), and at the last follow-up visit, the score was 1.5 (range: 1-5). At the last follow-up visit, the overall survival rate was $100 \%$. El Mabrouki et al. [12] also reported the improvement of the quality of life in patients with hemophilia after knee arthroplasty.

Compared with osteoarthritis, rheumatoid arthritis and other diseases, the prosthesis survival rate of HA after TKA is relatively low. Tagariello et al. [13] reported the incidence of TKA complications and revision 
rate up to 8 years in patients with hemophilia $(n=3,396)$ and vascular hemophilia $(n=1379)$, evaluated by using the national database, and compared with the matched cohort of patients with non-hemorrhagic diseases $(n=427,132$ and $n=394,657)$. They found that compared with the control group, hemophilia and von Willebrand disease were significantly associated with the incidence of infection, blood transfusion, medical complications and revision after TKA. The incidence of hemophilia, venous thromboembolism and blood transfusion was significantly higher in those patients than that of patients with vascular hemophilia and control group after TKA. In a study of 11 male patients ( 12 cases treated with THA in total), Colgan et al. [14] found that the average intraoperative blood loss was $502 \mathrm{ml}$ (100$1,250 \mathrm{ml})$, and the hemoglobin decreased by $3.25 \mathrm{~g} / \mathrm{dl}$ within $48 \mathrm{~h}$. Only one patient needed an intravenous infusion of two units of concentrated red blood cells, and there was no hematoma formation or other complications. Lin et al. [15] reported the results of a 30-year follow-up study of 17 cases treated with THA and 8 cases treated with TKA, and only 2 cases had prosthesis loosening. Botero et al. [16] studied 42 patients with hemophilia type A or B who underwent THA or TKA joint surgery $(71$ cases) and were followed up for more than 39 years. All patients used compression stockings for 6 weeks after surgery, 6 patients (10.5\%) used lower extremity sequential intermittent compression device, and 2 patients $(2.8 \%)$ received low molecular weight heparin. One patient (1.4\%) who received low molecular weight heparin treatment developed DVT of the lower extremity 10 days after hip arthroplasty for traumatic fracture, suggesting that hemophilia patients can undergo joint arthroplasty without conventional drugs for venous thromboembolism (VTE) to prevent the risk of thromboembolic events. This study supports the use of non-pharmacological DVT prevention measures, such as early foot pump exercise, lower extremity pneumatic pump. As DVT usually occurs in elderly patients, non-drug prevention of DVT may be more effective than prevention by drugs in hemophilic patients undergoing joint arthroplasty

In hemophilia patients, coagulation factor arthroplasty therapy requires the assistance of the Hematology Department, and the cost greatly exceeds the cost of prosthesis implantation[17] .Due to the high cost of coagulation factors and high perioperative consumption, a single infusion of coagulation factors in hemophilia patients is expected to solve as many problems as possible. In this study, homolateral THA plus TKA did not increase the overall risk, and the perioperative cost was significantly reduced. Also, Tonogai et al. [18] found that simultaneous bilateral surgery has advantages in reducing coagulation factor consumption, reducing hospital costs and shortening hospital stay. Repeated injections of coagulation factors are also associated with the risk of formation of inhibitory antibodies to coagulation factors in hemophilia patients. When a large number of coagulation factors are used, the risk of complications will increase. since, staged and multiple operations may increase the risk of the formation of inhibitory antibodies. Indeed, perioperative coagulation factor inhibitor plasty increases the incidence of complications, which is very dangerous and even fatal for patients[19] . The results of this study showed that simultaneous THA and TKA is a cost-effective surgical method. The consumption of coagulation factors, hospitalization expenses, length of stay, and various other human costs can be even lower than staged THA and TKA. With the improvement of living standards, hemophilia patients have higher requirements for quality of life and ability to resume work and other activities, thus joint 
arthroplasty cases will continue to increase, and the economic benefits of ipsilateral THA and TKA in the same period will be more significant. The operation sequence of this study is the THA operation first, followed by the TKA operation. The reasons are as follows: THA operation will affect the length of the lower limb, rotation center of femoral head, femoral valgus angle and force line, and then the TKA operation can be carried out according to the "index" after the THA operation. If the sequence is reversed, there may be the risk of early prosthesis failure due to the change of the TKA reference force line. It is helpful for patients to perform rehabilitation exercise and avoid the problem of mutual restriction of hip and knee joint activities, and establish a good foundation for contralateral limb diseases in the future. This study did not consider the simultaneous arthroplasty of more than three joints, as it will further increase the operation time, surgical bleeding, high risk of infection. Thus, we think that for such patients, simultaneous arthroplasty of two joints is more appropriate.

The limitation of this study lies in the small number of cases, the retrospective study design, the lack of control group and the short follow-up time. Therefore, we need indicators with larger sample size, longer follow-up time and better quality.

In conclusion, the authors believe that close cooperation and a joint management strategy with a hematology expert team is the key to ensure the safety of the operation, and long-term maintenance of coagulation factor / factor level is also needed to help prevent hip and knee joint bleeding after joint arthroplasty. On the premise of sufficient coagulation factor arthroplasty therapy, simultaneous ipsilateral THA and TKA have significant clinical efficacy in the treatment of advanced HA, which can significantly improve joint function, relieve pain and improve the quality of life. However, ipsilateral THA and TKA still face great challenges in the treatment of $\mathrm{HA}$, which requires the cooperation of joint surgeons and hematologists.

Typical case: Luo, male, 37 years old, suffered from repeated pain and swelling of the left hip and knees for 14 years. He was diagnosed as "hemophilia type A" at the age of 1 year and treatment was effective after transfusion of coagulation factor VIII. After repeated joint bleeding swelling and pain, the basic activity of factor VIII was $0.1 \%$, aPTT: $104 \mathrm{~s}$. At present, the treatment is on demand, 2,000 IU, and the exposure day is more than 150 days. Under the management of the MDT team, the left total hip arthroplasty and left total knee arthroplasty were performed at the same time.

Table 1. Preoperative and postoperative hip and knee function scores

\begin{tabular}{|lllll|}
\hline & Harris score & HSS score & Clinical KSS & Functional KSS \\
\hline Before operation & $16.8 \pm 4.4$ & $41.8 \pm 4.2$ & $35.6 \pm 10.8$ & $22.8 \pm 8.4$ \\
\hline Final follow-up & $77.6 \pm 7.1$ & $76.0 \pm 5.8$ & $79.2 \pm 6.9$ & $72.0 \pm 5.9$ \\
\hline P value & $<0.05$ & $<0.05$ & $<0.05$ & $<0.05$ \\
\hline
\end{tabular}

\section{Declarations}




\section{Ethics approval and consent to participate}

The experimental protocol was established, according to the ethical guidelines of the Helsinki Declaration and was approved by the Human Ethics Committee of Ethics Committee of the Second Affiliated Hospital of Chongqing Medical University. Written informed consent was obtained from individual or guardian participants.

\section{Consent for publication}

Not applicable.

\section{Availability of data and materials}

Not applicable.

\section{Competing interests}

Not applicable.

\section{Funding}

Not applicable.

\section{Authors' contributions}

Xu Peng, Shirong Chen, Xiangjun Chen and Xiaowei xie wrote the main manuscript text and Mao Nie prepared figures and table.

\section{Acknowledgements}

Not applicable.

\section{References}

[1] Timothy Matthews, Andrew Carr. Orthopaedic surgery and haemophilia. Current Orthopaedics, 2004, 18(5):345-356.

[2] A. Jandial, K.Mishra, R.Sandal, et al. Hemophilia in the developing world: transforming lives through international collaboration. Blood Advances, 2018, 2(30)39-41.

[3] Giuseppe Tagariello, Alfonso lorio, Elena Santagostino, et al. Comparison of the rates of joint arthroplasty in patients with severe factor VIII and IX deficiency: an index of different clinical severity of the 2 coagulation disorders. Blood, 2009, 23(114):779-784.

[4] Pier Mannuccio Mannucci, Massimo lacobelli. Progress in the contemporary management of hemophilia: The new issue of patient aging. European Journal of Internal Medicine, 2017,43:16-21. 
[5] E. Carlos Rodriguez-Merchan. Radiosynovectomy in haemophilia. Blood Reviews, 2019, 35:1-6.

[6] Christian Carulli, Irene Felici, Caterina Martini, et al. Total Hip Arthroplasty in Haemophilic Patients with Modern Cementless Implants. The Journal of Arthroplasty, 2015,30:1757-1760.

[7] Jerzy Mirosław Jaworski, Adam Zawojski, Joanna Zdziarska. Aloplasty of an ankylosed knee in a patient with severe haemophilia A. Acta Haematologica Polonica, 2017, 48(3):212-216.

[8] E. Carlos Rodriguez-Merchan. Orthopedic Management in Hemophilia: A Spanish Outlook. Seminars in Hematology, 2008, 45(2 Suppl 1):S58-63.

[9] Bhaveen H.Kapadia, Matthew R. Boylan ScB, Randa K. Elmallah, et al. Does Hemophilia Increase the Risk of Postoperative Blood Transfusion After Lower Extremity Total Joint Arthroplasty? The Journal of Arthroplasty, 2016,31(7):1578-1582.

[10] Sheng-Hao Wang, Chi-Hsiang Chung, Yeu-Chin Chen, et al. Does Hemophilia Increase Risk of Adverse Outcomes Following Total Hip and Knee Arthroplasty? A Propensity ScoreeMatched Analysis of a Nationwide, Population-Based Study. The Journal of Arthroplasty, 2019, 34(10):2329-2336.

[11] Liddle AD, Rodriguez-Merchan EC. Evidence-based management of the knee in hemophilia. JBJS Rev, 2017, 5(8):e12.

[12] B.El Mabrouki, D.Cherqaoui, L.Moustadraf, et al. Quality of life after double total knee arthroplasty on hemophilic arthropathy: Two case reports. Annals of Physical and Rehabilitation Medicine, 2011, 54(1):e99.

[13] Jourdan M.Cancienne, Brian C.Werner, James A.Browne. Complications After TKA in Patients With Hemophilia or Von Willebrand's Disease. The Journal of Arthroplasty, 2015, 30(12):2285-2290.

[14] Grainne Colgan, Joseph F.Baker, N.Donlon, et al. Total hip arthroplasty in patients with haemophilia What are the risks of bleeding in the immediate peri-operative period? Journal of Orthopaedics, 2016, 13(4):389-393.

[15] CHU Yang Lin, Haowei Sun. Immediate and Long-Term Outcomes Following Orthopedic Procedures in Patients with Hemophilia: A Three-Decade Retrospective Review. Blood, 2019,134(Supplement_1):5874.

[16] Juliana Perez Botero, Daniel B. Spoon, Mrinal S. Patnaik, et al. Incidence of symptomatic venous thromboembolism in patients with hemophilia undergoing joint arthroplasty surgery:A retrospective study. Thrombosis Research, 2015,135(1):109-113.

[17] Peter Salaj, Miroslav Penk, Petr Smejkal, et al. Economic analysis of recombinant activated factor VII versus plasma-derived activated prothrombin complex concentrate in mild to moderate bleeds: Haemophilia registry data from the Czech Republic. Thrombosis Research, 2012,129(5):e233-e237. 
[18] Ichiro Tonogai, Koichi Sairyo. A case of arthroscopic ankle arthrodesis for hemophilic arthropathy of the bilateral ankles. International Journal of Surgery Case Reports, 2020, 74:251-256.

\section{Figures}
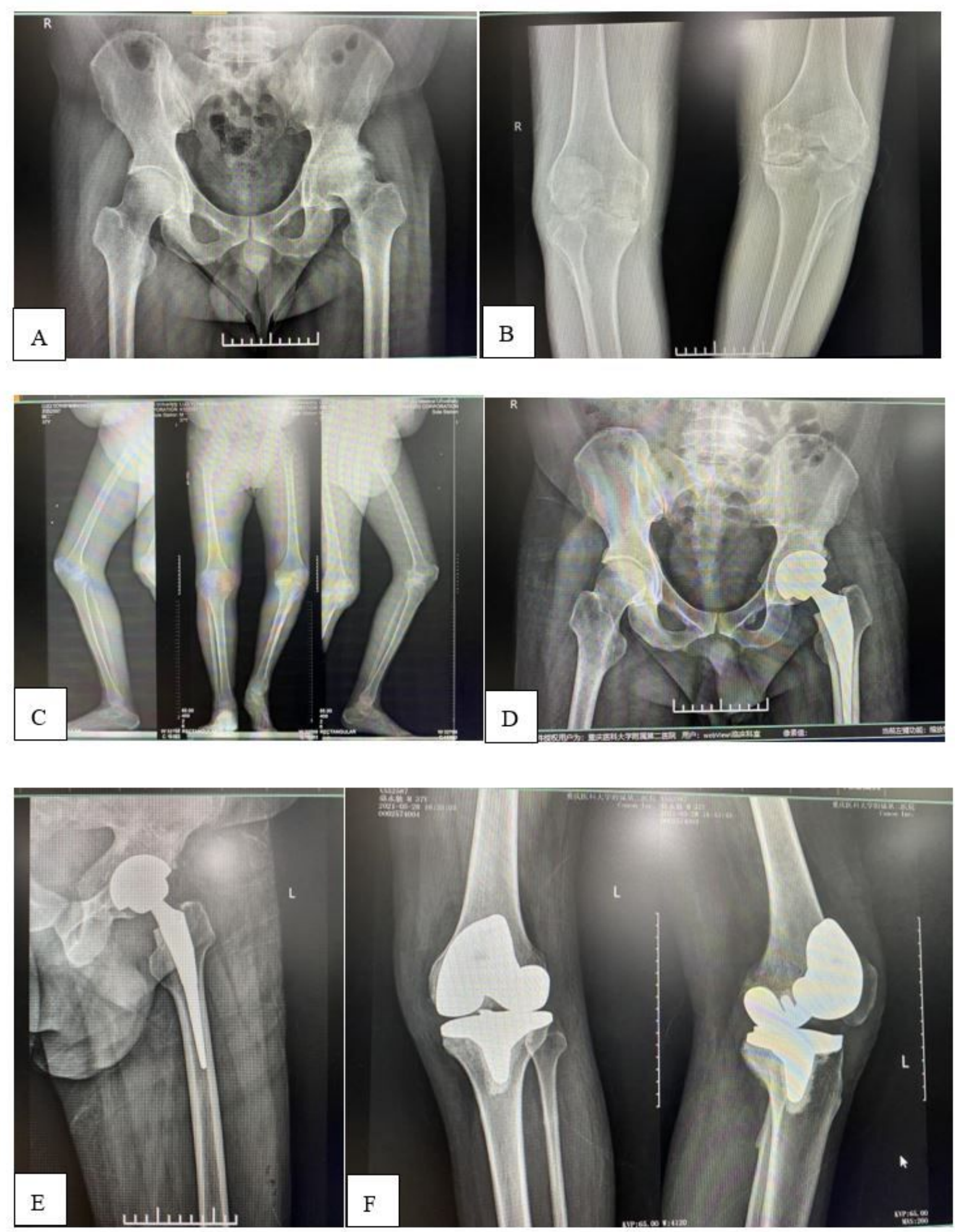

Figure 1 
Perioperative X-ray films of typical cases $A$ shows a preoperative pelvic plain film; B displays a anteroposterior and lateral $\mathrm{X}$-ray image of both knees before operation; $\mathrm{C}$ shows a preoperative full length image of both lower limbs; D shows a postoperative pelvic plain image; E displays a lateral X-ray of the left hip after operation; $F$ shows the $X$-ray of the left knee after operation. 